University of New Orleans

ScholarWorks@UNO

$1-10-2005$

\title{
Single-layer-coated beam splitters for the division-of-amplitude photopolarimeter
}

\author{
Rasheed M.A. Azzam \\ University of New Orleans, razzam@uno.edu \\ Faisal F. Sudradjat
}

Follow this and additional works at: https://scholarworks.uno.edu/ee_facpubs

Part of the Electrical and Electronics Commons

\section{Recommended Citation}

Rasheed M. A. Azzam and Faisal F. Sudradjat, "Single-layer-coated beam splitters for the division-ofamplitude photopolarimeter," Appl. Opt. 44, 190-196 (2005)

This Article is brought to you for free and open access by the Department of Electrical Engineering at ScholarWorks@UNO. It has been accepted for inclusion in Electrical Engineering Faculty Publications by an authorized administrator of ScholarWorks@UNO. For more information, please contact scholarworks@uno.edu. 


\title{
Single-layer-coated beam splitters for the division-of-amplitude photopolarimeter
}

\author{
Rasheed M. A. Azzam and Faisal F. Sudradjat
}

\begin{abstract}
A design procedure is presented for a near-optimal, single-layer-coated prism beam splitter that serves as the key optical element of the division-of-amplitude photopolarimeter (DOAP). For given film and substrate refractive indices, the angle of incidence and film thickness are selected such that the ellipsometric differential phase shifts in reflection and transmission $\Delta_{r}$ and $\Delta_{t}$ differ by $\pm \pi / 2$, and the normalized determinant of the instrument matrix is maximized. The best results are obtained by using highindex films on low-index substrates. This is illustrated by examples of $\mathrm{ZnS}$ and GaP films on silica prisms in the visible and $\mathrm{Si}, \mathrm{Ge}$, and $\mathrm{PbTe}$ films on Irtran 1 substrates in the infrared. A $16^{\circ} \mathrm{Si}$-prism DOAP beam splitter at the $1.55-\mu \mathrm{m}$ lightwave-communications wavelength is also presented. It uses a $163-\mathrm{nm}^{\mathrm{SiO}_{2}}$ coating on the entrance face to satisfy the optimum delta condition at $73^{\circ}$ incidence, and the determinant of the instrument matrix is $78.23 \%$ of its theoretical maximum. The exit face of the Si prism is antireflection coated with a $208-\mathrm{nm} \mathrm{Si}_{3} \mathrm{~N}_{4}$ film. (C) 2005 Optical Society of America

OCIS codes: $120.2130,120.5410,230.1360,240.0310,260.5430$.
\end{abstract}

\section{Introduction}

The most general state of partial elliptical polarization of quasi-monochromatic light is described by the four Stokes parameters $S_{0}, S_{1}, S_{2}$, and $S_{3}$. These parameters are often lumped in a $4 \times 1$ Stokes vector

$$
\mathbf{S}=\left[S_{0} S_{1} S_{2} S_{3}\right]^{\mathrm{t}},
$$

where $\mathrm{t}$ indicates the matrix transpose. Numerous optical polarimeters have been designed ${ }^{1-3}$ for measuring some or all of the components of $\mathbf{S}$.

In the division-of-amplitude photopolarimeter ${ }^{4,5}$ (DOAP), Fig. 1, the collimated incident light beam (i) whose Stokes parameters are to be measured is split into four separate beams with a beam splitter (BS) and two Wollaston prisms WP1 and WP2 (or equivalent polarizing BS's). Linear detection of the light fluxes of the four component beams by photodetectors $\mathrm{D}_{0}, \mathrm{D}_{1}, \mathrm{D}_{2}$, and $\mathrm{D}_{3}$ yields an electrical output signal vector

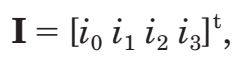

which is linearly related to the Stokes vector $\mathbf{S}$ of

R. Azzam (razzam@uno.edu) and F. Sudradjat are with the Electrical Engineering Department, University of New Orleans, New Orleans, Louisiana 70148.

Received 26 April 2004; revised manuscript received 28 September 2004; accepted 29 September 2004.

0003-6935/05/020190-07\$15.00/0

(C) 2005 Optical Society of America incident light by

$$
\mathbf{I}=\mathbf{A S} .
$$

From Eq. (3) the unknown polarization vector $\mathbf{S}$ is completely recovered from the measured signal vector $\mathbf{I}$ by

$$
\mathbf{S}=\mathbf{A}^{-1} \mathbf{I}
$$

provided that the instrument matrix $\mathbf{A}$ (which is determined by calibration ${ }^{6}$ ) is nonsingular, so that its inverse $\mathbf{A}^{-1}$ exists. Several DOAP instruments have been constructed and applied to reflection and scattering Mueller-matrix ellipsometry. ${ }^{7-11}$ Because the DOAP uses no moving parts or modulators, and generates four linearly independent projections of the unknown Stokes vector of incident light simultaneously (via four independent parallel channels), it is capable of fast and complete measurement of light polarization or scattering matrices under dynamic conditions.

When the Wollaston prisms are oriented at $\pm 45^{\circ}$ to filter the orthogonal linear polarization components of the reflected (r) and transmitted (t) light along the bisectors of the directions parallel $(p)$ and perpendicular $(s)$ to the plane of incidence at the BS, the determinant of the instrument matrix becomes ${ }^{5}$

$$
\begin{aligned}
\operatorname{det} A= & (R T)^{2} \sin 2 \psi_{r} \sin 2 \psi_{t}\left(\cos 2 \psi_{r}-\cos 2 \psi_{t}\right) \\
& \times \sin \left(\Delta_{r}-\Delta_{t}\right) .
\end{aligned}
$$




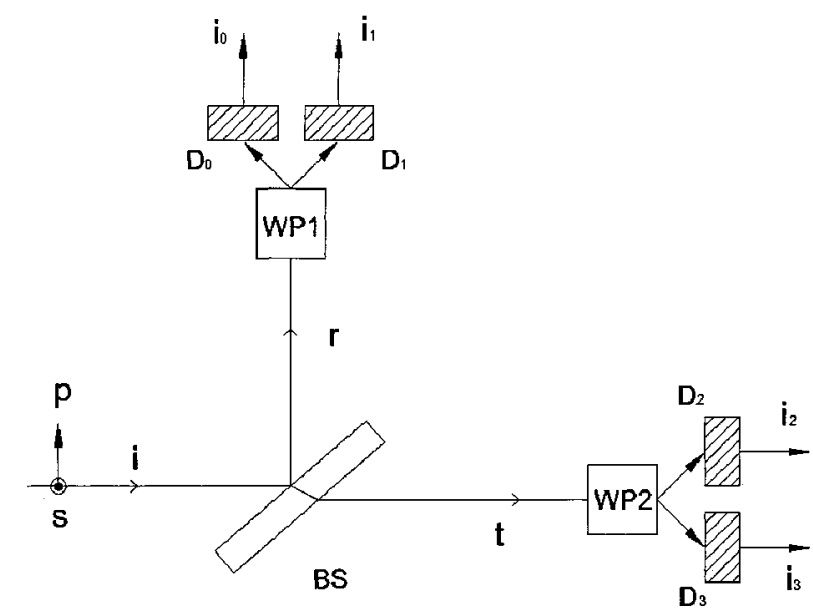

Fig. 1. Division-of-amplitude photopolarimeter (DOAP). BS, beam splitter to be designed; WP1, WP2, Wollaston prisms; $\mathrm{D}_{0}, \mathrm{D}_{1}, \mathrm{D}_{2} \mathrm{D}_{3}$, linear photodetectors that generate output electrical signals $\mathrm{i}_{0}, \mathrm{i}_{1}, \mathrm{i}_{2}$, and $\mathrm{i}_{3}$, respectively; $p$ and $s$ linear polarization directions parallel and perpendicular to the plane of incidence at $\mathrm{BS}$, respectively.

In Eq. (5) $R$ and $T$ are the BS intensity reflectance and transmittance for incident unpolarized light, and $\left(\psi_{r}, \Delta_{r}\right)$ and $\left(\psi_{t}, \Delta_{t}\right)$ are the reflection and transmission ellipsometric parameters ${ }^{12}$ of the BS. A constant premultiplier, proportional to the product of the photoelectric sensitivities of the four detectors, has been dropped from the right-hand side of Eq. (5).

Optimum optical parameters for the BS are those that make the instrument matrix as far from singular as possible by maximizing the absolute value of the determinant of Eq. (5). For all-dielectric (nonabsorbing) $\mathrm{BSs}$, the optimum parameters of the BS are given by ${ }^{13}$

$$
\begin{aligned}
\Delta_{r}-\Delta_{t} & = \pm \pi / 2, \\
R & =T=0.5, \\
\left(\psi_{r}, \psi_{t}\right) & =\left(\frac{1}{2} \arccos ( \pm 1 / \sqrt{3}), 90^{\circ}-\psi_{r}\right) \\
& =\left(27.368^{\circ}, 62.632^{\circ}\right) \text { or }\left(62.632^{\circ}, 27.368^{\circ}\right) .
\end{aligned}
$$

When the optimum parameters of Eqs. (6)-(8) are substituted into Eq. (5), the maximum absolute value of the determinant is obtained:

$$
|\operatorname{det} A|_{\max }=\sqrt{3} / 36=0.0481 \text {. }
$$

The extent to which a given BS meets conditions (6)-(8) is determined by the normalized determinant

$$
|\operatorname{det} A|_{\text {norm }}=|\operatorname{det} A| /|\operatorname{det} A|_{\max } \text {, }
$$

which should be as close to 1 as possible.

The simplest possible DOAP BS consists of a di-

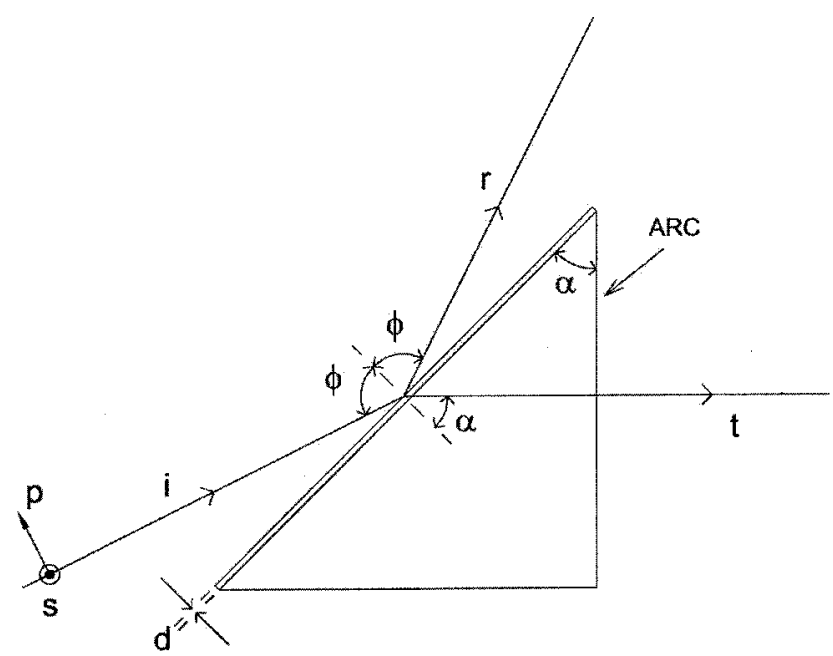

Fig. 2. Prism BS for DOAP. The incident collimated light beam i, whose polarization is to be measured, strikes the coated, beamsplitting entrance face of the prism at an angle $\phi$. The prism angle $\alpha$ equals the angle of refraction in the prism, so that the transmitted beam is normal to the (antireflection-coated) exit face of the prism. The angular separation between the reflected $(r)$ and the transmitted (t) beams is $\pi-(\phi+\alpha)$.

electric substrate of refractive index $n_{2}$ that is coated by a transparent thin film of refractive index $n_{1}$ and thickness $d$. To avoid multiple internal reflections inside a parallel slab, we propose the prism design shown in Fig. 2. Collimated light of wavelength $\lambda$ (whose polarization is to be measured) is incident on the coated, beam-splitting entrance face of the prism at an angle $\phi$. The prism angle $\alpha$ is equal to the angle of refraction in the prism, so that the transmitted beam is normal to the (antireflection-coated) exit face of the prism. The angular separation between the reflected $(\mathrm{r})$ and transmitted $(\mathrm{t})$ beams is $\pi-(\phi$ $+\alpha)$. Because we assume collimated incident light, the coated prism BS is intended mainly for nonimaging polarimeters.

Previously proposed film $\left(n_{1}\right)$ /substrate $\left(n_{2}\right) \mathrm{BSs}$ for the $\mathrm{DOAP}^{5,7}$ include $\mathrm{MgF}_{2}(1.38) / \mathrm{ZnS}(2.30)$ and $\mathrm{TiO}_{2}$ (2.08) $/ \mathrm{SiO}_{2}(1.52)$ at $\lambda=633 \mathrm{~nm}$ and $\phi=70^{\circ}$. From the published data ${ }^{5,7}$ on these two BSs, the normalized determinant $|\operatorname{det} A|_{\text {norm }}$ is calculated to be 0.3546 and 0.4271 , respectively, which is less than optimal. A better infrared design ${ }^{5}$ uses a $\mathrm{ThF}_{2}$ (1.35)/Ge (4.00) system at $\lambda=10.6 \mu \mathrm{m}$ and $\phi$ $=70^{\circ}$, for which $|\operatorname{det} A|_{\text {norm }}=0.6742$.

In this paper, we present a systematic approach for the design of an all-transparent film-substrate BS for the DOAP with near optimal performance (i.e., $|\operatorname{det} A|_{\text {norm }}$ is essentially 1$)$. The design procedure is described in Section 2. Results for several BSs that use high-index films on low-index substrates in the visible and infrared are given, and the angular and spectral response of one selected design are presented in Section 3. BSs that use low-index films on highindex substrates are discussed in Section 4. Finally, Section 5 gives a brief summary of the paper. 


\section{Design of Single-Layer-Coated Beam Splitter for the DOAP}

The complex-amplitude reflection and transmission coefficients of the coated surface are given by ${ }^{12}$

$$
\begin{gathered}
R_{\nu}=\left(r_{01 v}+r_{12 v} X\right) /\left(1+r_{01 v} r_{12 v} X\right), \\
T_{\nu}=\left(t_{01 v} t_{12 v} X^{1 / 2}\right) /\left(1+r_{01 v} r_{12 v} X\right) .
\end{gathered}
$$

In Eqs. (11) $r_{i j v}, t_{i j v}$ are the Fresnel reflection and transmission coefficients of the $i j$ interface for the $v$ polarization $(v=p, s)$, and

$$
\begin{aligned}
X & =\exp (-j \theta), \\
\theta & =2 \pi d / D, \\
D & =(\lambda / 2)\left(n_{1}{ }^{2}-\sin ^{2} \phi\right)^{-1 / 2} .
\end{aligned}
$$

We assume that the medium of incidence is air with refractive index $n_{0}=1$. The ellipsometric parameters of the coated BS surface in reflection and transmission are determined by ${ }^{12}$

$$
\begin{gathered}
\rho_{r}=R_{p} / R_{s}=\tan \psi_{r} \exp \left(j \Delta_{r}\right), \\
\rho_{t}=T_{p} / T_{s}=\tan \psi_{t} \exp \left(j \Delta_{t}\right) .
\end{gathered}
$$

A critical parameter in the operation of the DOAP is the ratio

$$
\begin{aligned}
& \Gamma=\rho_{r} / \rho_{t}=|\Gamma| \exp (j \gamma), \\
& \gamma=\Delta_{r}-\Delta_{t} .
\end{aligned}
$$

From Eqs. (11), (14), and (15) we obtain

$$
\Gamma=b\left(1+B_{p} X\right) /\left(1+B_{s} X\right),
$$

where

$$
\begin{aligned}
b & =\left(r_{01 p} / r_{01 s}\right)\left(t_{01 s} / t_{01 p}\right)\left(t_{12 s} / t_{12 p}\right), \\
B_{p} & =\left(r_{12 p} / r_{01 p}\right), \\
B_{s} & =\left(r_{12 s} / r_{01 s}\right) .
\end{aligned}
$$

Because all media are transparent, and total internal reflection does not occur, all of the quantities that appear in Eqs. (17) are real. Consequently, the angle $\gamma=\Delta_{r}-\Delta_{t}$ of $\Gamma$ is obtained from Eqs. (12), (15), and (16) as

$$
\tan \gamma=\left(B_{p}-B_{s}\right) \sin \theta /\left[\left(1+B_{p} B_{s}\right)+\left(B_{p}+B_{s}\right) \cos \theta\right] .
$$

To satisfy the optimum polarimetric requirement concerning the phase shifts introduced by the DOAP $\mathrm{BS}$ in reflection and transmission, Eq. (6), the denom- inator of the right-hand side of Eq. (18) must be zero. This gives

$$
\cos \theta=-\left(1+B_{p} B_{s}\right) /\left(B_{p}+B_{s}\right) .
$$

From Eqs. (17) and (19) we obtain

$$
\begin{aligned}
\cos \theta & =f\left(\phi, n_{1}, n_{2}\right) \\
& =-\left(r_{01 p} r_{01 s}+r_{12 p} r_{12 s}\right) /\left(r_{01 p} r_{12 s}+r_{01 s} r_{12 p}\right) .
\end{aligned}
$$

Equation (20) has a valid solution for $\theta$ if $-1 \leq f$ $\leq+1$.

Based on the above analysis, the design procedure consists of the following steps:

(1) For a given film-substrate system at a given wavelength $\lambda$ (i.e., for given $n_{1}, n_{2}$ ), all possible solutions of Eq. (20) are determined as $\phi$ is changed as a parameter in steps of $\leq 1^{\circ}$. Each solution represents a design that satisfies the optimum phase condition of Eq. (6).

(2) The normalized determinant, Eq. (10), is calculated and plotted as a function of $\phi$ within the solution range. The optimum operating angle is that at which $|\operatorname{det} A|_{\text {norm }}$ is maximum.

(3) When the maximum value of $|\operatorname{det} A|_{\text {norm }}$ is sufficiently near 1 , the remaining optimum conditions of Eqs. (7) and (8) are approximately satisfied (to within a small error), and a near-optimal design is achieved.

\section{DOAP Beam Splitters with High-Index Film on Low-Index Substrate}

For a high-index film on a low-index substrate $\left(n_{1}>n_{2}\right)$, the lowest angle of incidence $\phi_{1}$ at which Eq. (20) has a solution is the Brewster angle of the ambient-substrate (02) interface,

$$
\phi_{1}=\phi_{B 02}=\arctan n_{2} .
$$

At this angle, we have $r_{01 p}+r_{12 p}=0$, hence

$$
r_{01 p}=-r_{12 p} .
$$

Substitution of Eq. (22) into Eq. (20) gives

$$
\cos \theta=+1
$$

hence $\theta=0, \theta=2 \pi, X=1$, which correspond to $d$ $=0$ or $d=D$, i.e., no coating or an absentee layer.

The upper limit $\phi_{2}$ of the range of $\phi$ for which Eq. (20) has a solution corresponds to

$$
r_{01 p}=r_{12 p}
$$

Substitution of Eq. (24) into Eq. (20) shows that

$$
\cos \theta=-1
$$

hence $\theta=\pi, X=-1, d=D / 2$, i.e., the layer is of 


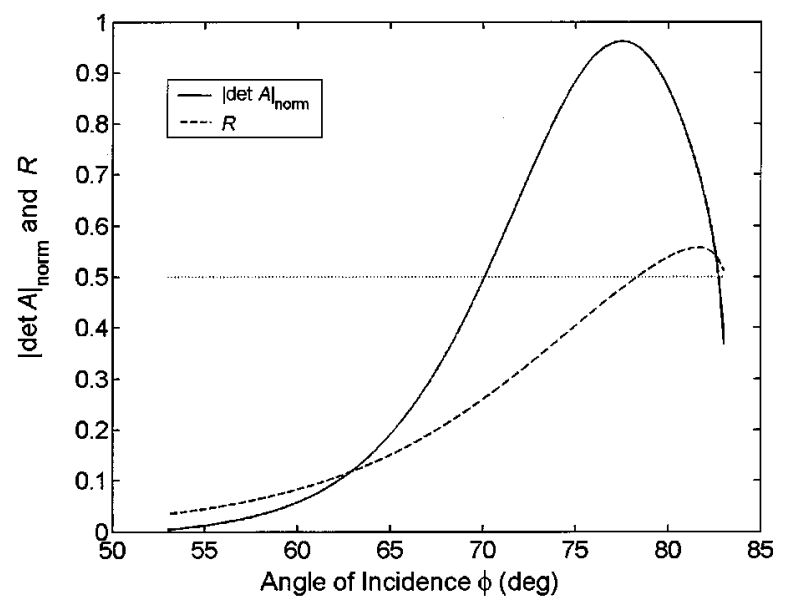

Fig. 3. Normalized determinant, $|\operatorname{det} A|_{\text {norm }}$, and unpolarizedlight reflectance $R$ plotted as functions of the angle of incidence $\phi$ for BSs that consist of a Ge film $\left(n_{1}=4.00\right)$ on an Irtran 1 substrate $\left(n_{2}=1.30\right)$ at wavelength $\lambda=6.75 \mu \mathrm{m}$ such that the phase condition of Eq. (6) is exactly satisfied at each angle. The best design is obtained at $\phi=78^{\circ}$, where $\left(|\operatorname{det} A|_{\text {norm }}\right)_{\max }=0.9583$, and the remaining optimum conditions of Eqs. (7) and (8) are nearly satisfied.

quarter-wave optical thickness at oblique incidence. From Eq. (24) and the Fresnel formulas, ${ }^{12}$ we obtain the following equation for $\phi_{2}$ :

$$
n_{1}^{4} \cos \phi_{2}\left(n_{2}^{2}-\sin ^{2} \phi_{2}\right)^{1 / 2}=n_{2}^{2}\left(n_{1}^{2}-\sin ^{2} \phi_{2}\right) .
$$

Squaring both sides of Eq. (26) leads to a quadratic equation in $\sin ^{2} \phi_{2}$. At the limiting angles $\phi_{1}$ and $\phi_{2}$ the $p$ polarization is suppressed on reflection, and

$R_{p}=0, \quad \rho_{r}=0, \quad \psi_{r}=0, \quad \operatorname{det} A=0$.

As a specific example, consider a Ge film $\left(n_{1}\right.$ $=4.00)$ on an Irtran 1 substrate $\left(n_{2}=1.30\right)$ at wavelength $\lambda=6.75 \mu \mathrm{m} .{ }^{14}$ From Eqs. (21) and (26), we obtain $\phi_{1}=52.431^{\circ}$ and $\phi_{2}=83.216^{\circ}$. Figure 3 shows $|\operatorname{det} A|_{\text {norm }}$ and $R$ plotted as functions of $\phi$ in the range $\phi_{1}<\phi<\phi_{2}$. A near-optimum design is obtained at $\phi=78^{\circ}$, where

$$
\begin{gathered}
\alpha=48.80^{\circ}, \quad \theta=54.3027^{\circ}, \\
\Delta_{r}-\Delta_{t}=-90^{\circ}, \\
R=0.4916, \quad T=0.5084, \\
\psi_{r}=23.537^{\circ}, \quad \psi_{t}=65.599^{\circ}, \\
|\operatorname{det} A|_{\text {norm }}=0.9583 .
\end{gathered}
$$

The above results show that Eq. (6) is satisfied exactly, and Eq. (7) is satisfied to within $<1 \%$. However, according to Eq. (8), $\psi_{r}$ and $\psi_{t}$ are off from their optimum values by $14 \%$ and $5 \%$, respectively. The thick-

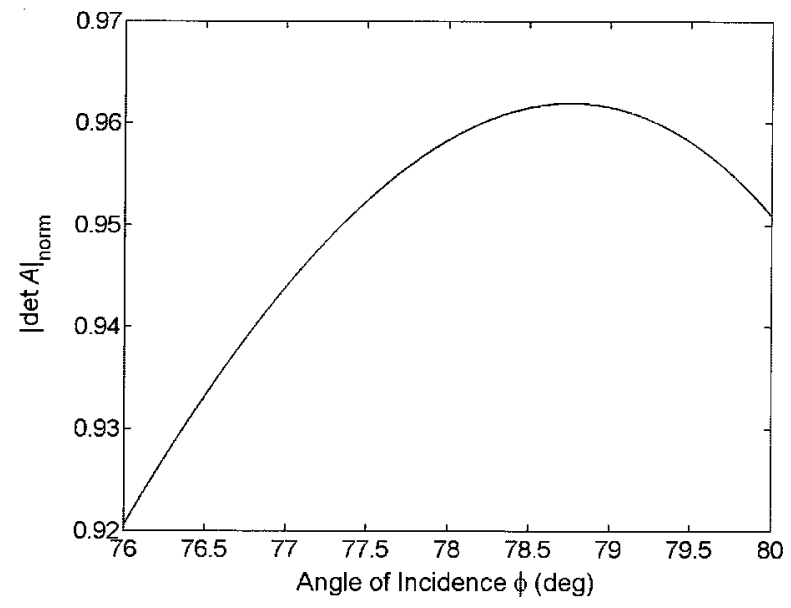

Fig. 4. Normalized determinant, $|\operatorname{det} A|_{\text {norm }}$ as a function of angle of incidence $\phi$ for a DOAP BS that consists of a $131.3-\mathrm{nm} \mathrm{Ge}$ thin film on an Irtran 1 substrate at wavelength $\lambda=6.75 \mu \mathrm{m}$. Note that $|\operatorname{det} A|_{\text {norm }}$ remains near its maximum for $76 \leq \phi \leq 80^{\circ}$.

ness of the Ge film as a fraction of the thickness period is

$$
d / D=\theta / 2 \pi=0.1508 .
$$

This differs from the eighth-wave thickness $(d / D$ $=0.25$ ) that was intuitively suggested previously. ${ }^{5}$ From Eqs. (13), we also obtain the metric thickness of the Ge film,

$$
d=131.26 \mathrm{~nm} .
$$

The 131.3-nm Ge thin-film coating on the Irtran 1 substrate functions well as a DOAP BS over a range of incidence angles and wavelengths. This is illustrated in Figs. 4 and 5, which show the $|\operatorname{det} A|_{\text {norm }}$ as a function of $\phi$ and $\lambda$, respectively.

Figure 4 shows that $|\operatorname{det} A|_{\text {norm }}>0.92$ for 76 $\leq \phi \leq 80^{\circ}$. When the metric film thickness is kept constant $(d=131.26 \mathrm{~nm})$ and the angle of incidence is changed, the optimum delta condition of Eq. (6) is no longer satisfied, and all of the BS parameters are shifted from the values listed in Eqs. (28). In Fig. 4 $|\operatorname{det} A|_{\text {norm }}$ reaches a new maximum at $\phi=78.8^{\circ}$, which is slightly higher than that obtained under the conditions of constrained optimization shown in Fig. 3.

Figure 5 shows that $|\operatorname{det} A|_{\text {norm }}$ increases as the wavelength is increased over the range $5.75 \leq \lambda$ $\leq 7.75 \mu \mathrm{m}$. (The dispersion of Ge and Irtran 1 is accounted for ${ }^{14,15}$ in Fig. 5.) For accurate spectroscopic polarimetry, the DOAP should be calibrated to determine the instrument matrix $\mathbf{A}(\phi, \lambda)$ as a function of the operating incidence angle and wavelength.

In Fig. 6 we present results for $|\operatorname{det} A|_{\text {norm }}$ as a function of $\phi$ for five different film-substrate systems: (a) $\mathrm{ZnS}(2.352) / \mathrm{SiO}_{2}$ (1.457) at $\lambda=633 \mathrm{~nm}$, (b) $\mathrm{GaP}(3.308) / \mathrm{SiO}_{2}(1.457)$ at $\lambda=633 \mathrm{~nm}$, (c) $\mathrm{Si}$ (3.432) $/ \mathrm{SiO}_{2}(1.419)$ at $\lambda=3 \mu \mathrm{m},(\mathrm{d}) \mathrm{Ge}(4.00) / \operatorname{Irtran} 1$ 


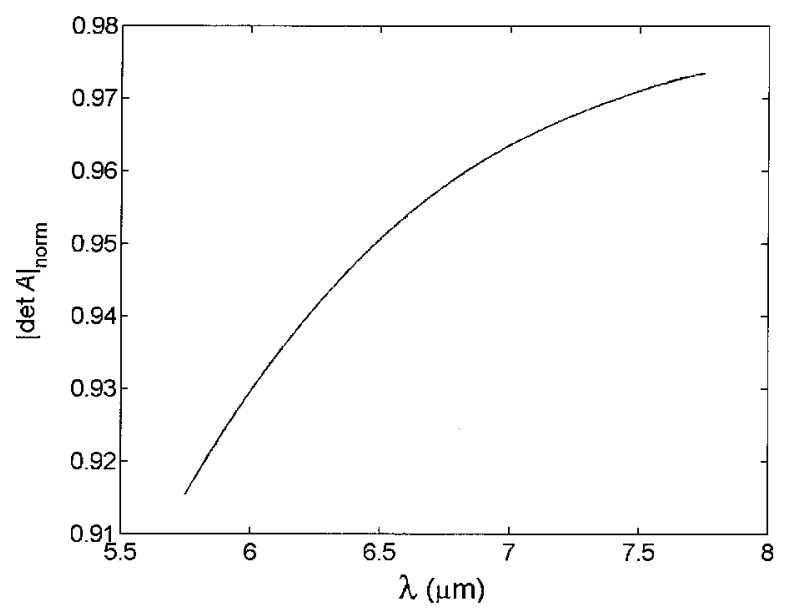

Fig. 5. Normalized determinant $|\operatorname{det} A|_{\text {norm }}$ as a function of wavelength $\lambda$ for a DOAP BS that consists of a 131.3-nm Ge thin film on an Irtran 1 substrate at an angle of incidence $\phi=78^{\circ}$. Note that $|\operatorname{det} A|_{\text {norm }}$ increases as $\lambda$ increases in the spectral range $5.75 \leq \lambda \leq 7.75 \mu \mathrm{m}$.

(1.30) at $\lambda=6.75 \mu \mathrm{m}$, and (e) PbTe (5.655)/Irtran 1 (1.227) at $\lambda=9 \mu \mathrm{m}$. The refractive indices of all materials are obtained from Refs. 14 and 15. As the film refractive index $n_{1}$ increases, the peak of $|\operatorname{det} A|_{\text {norm }}$ increases and its location is shifted toward higher angles. For the PbTe/Irtran 1 system the highest normalized determinant $\left(|\operatorname{det} A|_{\text {norm }}\right)_{\max }$ $=0.99$ occurs at $\phi \approx 79^{\circ}$ and $d / D=0.0883$. Because of the low refractive index of Irtran 1 at $\lambda=9 \mu \mathrm{m}$, the reflection loss at the exit face of the prism $(\alpha$ $=53.132^{\circ}$ ) without an antireflection coating is only $1 \%$.

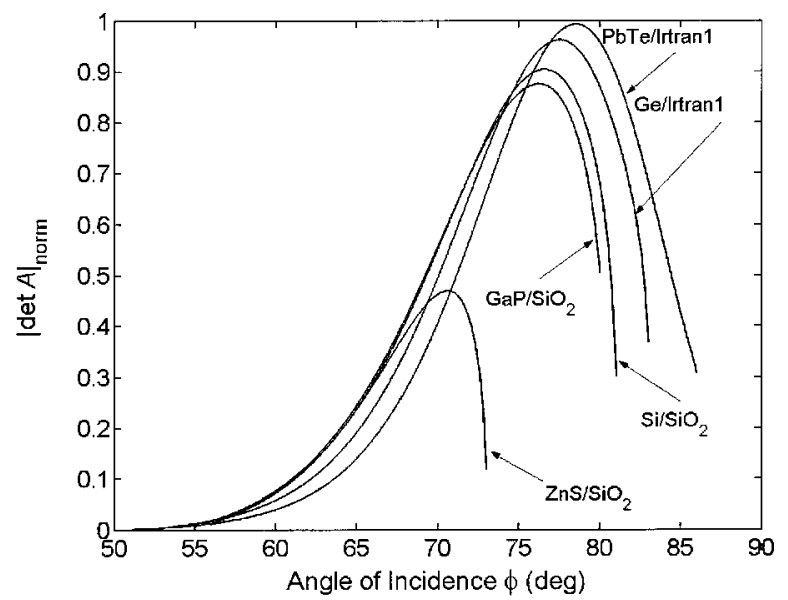

Fig. 6. Normalized determinant $|\operatorname{det} A|_{\text {norm }}$ as a function of $\phi$ for five different film-substrate systems: (a) $\mathrm{ZnS}(2.352) / \mathrm{SiO}_{2}$ (1.457) at $\lambda=633 \mathrm{~nm}$, (b) GaP (3.308) $/ \mathrm{SiO}_{2}(1.457)$ at $\lambda=633 \mathrm{~nm}$, (c) $\mathrm{Si}$ (3.432) $/ \mathrm{SiO}_{2}$ (1.419) at $\lambda=3 \mu \mathrm{m},(\mathrm{d}) \mathrm{Ge}(4.00) / \operatorname{Irtran} 1$ (1.30) at $\lambda=6.75 \mu \mathrm{m}$, and (e) PbTe (5.655)/Irtran 1 (1.227) at $\lambda=9 \mu \mathrm{m}$. The refractive indices of all materials are obtained from Refs. 14 and 15. As the film refractive index $n_{1}$ increases, the peak of $|\operatorname{det} A|_{\text {norm }}$ increases, and its location is shifted toward higher angles.

\section{DOAP Beam Splitters with Low-Index Film on High-Index Substrate}

We also consider the case of a low-index film on a high-index substrate $\left(n_{1}<n_{2}\right)$. The lowest angle of incidence $\phi_{1}$ at which the delta criterion [Eqs. (6) and (20)] is satisfied is determined by

$$
r_{01 s}=r_{12 s} .
$$

Substitution of Eq. (31) into Eq. (20) gives

$$
\cos \theta=-1
$$

hence $\theta=\pi, X=-1$, and $d=D / 2$, i.e., the coating is of quarter-wave optical thickness at oblique incidence. This coating suppresses the $s$ polarization on reflection, so that

$$
R_{s}=0, \quad \rho_{r}=\infty, \quad \psi_{r}=90^{\circ}, \quad \operatorname{det} A=0 .
$$

From Eq. (31) and the Fresnel formulas, ${ }^{12}$ the angle $\phi_{1}$ is given by

$$
\tan ^{2} \phi_{1}=\left(n_{2}^{2}-n_{1}^{4}\right) /\left(n_{1}^{2}-1\right)^{2} .
$$

The upper limit $\phi_{2}$ of the range of $\phi$ for which Eq. (20) has a solution corresponds to the Brewster angle of the ambient-substrate (02) interface:

$$
\phi_{2}=\phi_{B 02}=\arctan n_{2} .
$$

As a specific example, we take a $\mathrm{Si}$ prism that is oxidized on its beam-splitting face. For this $\mathrm{SiO}_{2}$ (1.444)/Si (3.478) system $^{15}$ at the lightwavecommunications wavelength $\lambda=1.55 \mu \mathrm{m}$, Eqs. (34) and (35) give $\phi_{1}=68.703^{\circ}$ and $\phi_{2}=73.959^{\circ}$. When $|\operatorname{det} A|_{\text {norm }}$ is calculated as a function of $\phi$ in the range $\phi_{1}<\phi<\phi_{2}$, we obtain $|\operatorname{det} A|_{\text {norm }}=0.7823$, which is near maximum, at $\phi=73^{\circ}$. This indicates good performance at a lower angle of incidence. Other parameters for this $\mathrm{SiO}_{2} / \mathrm{Si}$ prism DOAP BS are given by

$$
\begin{gathered}
\alpha=15.96^{\circ}, \\
\theta=81.817^{\circ}, \quad d / D=0.2273, \\
\Delta_{r}-\Delta_{t}=90^{\circ}, \\
R=0.363, \quad T=0.637, \\
\psi_{r}=26.955^{\circ}, \quad \psi_{t}=54.809^{\circ} .
\end{gathered}
$$

The refractive index of a quarter-wave, single-layer, antireflection coating at the exit face of this prism is $n_{1}=\sqrt{n_{2}}=1.865$, which falls within the range of values of the refractive index of nonstoichiometric silicon nitride. ${ }^{16}$ The thickness of this antireflection 


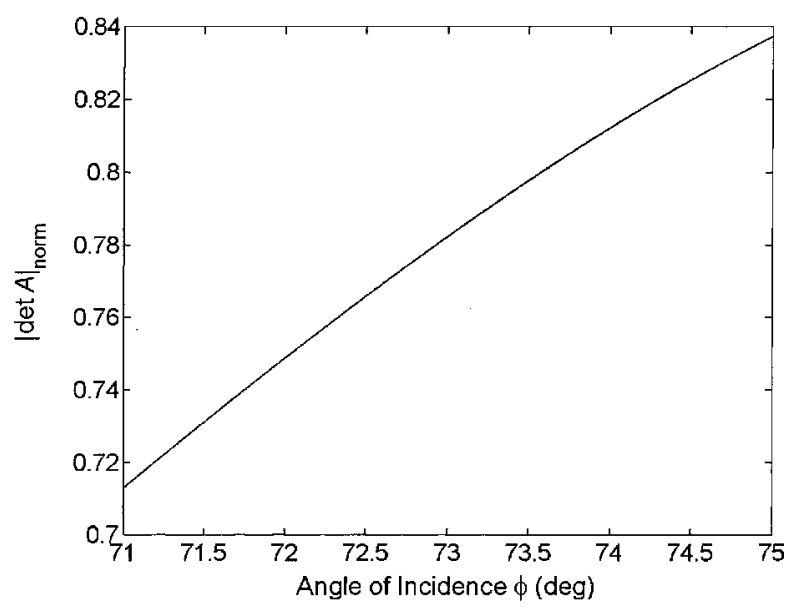

Fig. 7. Normalized determinant, $|\operatorname{det} A|_{\text {norm }}$ as a function of angle of incidence $\phi$ for a DOAP BS that consists of a $163-\mathrm{nm} \mathrm{SiO}_{2}$ thin film on a Si substrate at wavelength $\lambda=1.55 \mu \mathrm{m}$. $|\operatorname{det} A|_{\text {norm }}$ increases monotonically with $\phi$ in the range $71 \leq \phi \leq 75^{\circ}$.

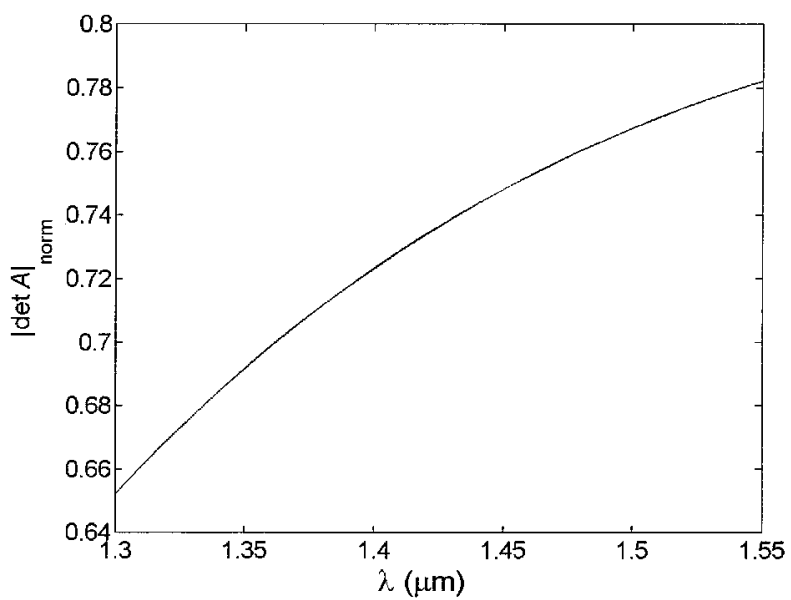

Fig. 8. Normalized determinant, $|\operatorname{det} A|_{\text {norm }}$ as a function of wavelength $\lambda$ for a DOAP BS that consists of a $163-\mathrm{nm} \mathrm{SiO}_{2}$ thin film on a Si substrate at an angle of incidence $\phi=73^{\circ}$. $|\operatorname{det} A|_{\text {norm }}$ increases monotonically with $\lambda$ in the spectral range $1.30 \leq \lambda$ $\leq 1.55 \mu \mathrm{m}$.

coating is $207.8 \mathrm{~nm}$. Use of Si-related thin films on the entrance and exit faces of the Si prism makes this BS design particularly attractive.

For completeness, Figs. 7 and 8 show the normalized determinant $|\operatorname{det} A|_{\text {norm }}$ as a function of $\phi$ (at the design wavelength $\lambda=1.55 \mu \mathrm{m}$ ) and as a function of $\lambda$ (at the design angle $\phi=73^{\circ}$ ), respectively, for the $\mathrm{SiO}_{2} / \mathrm{Si}$ prism DOAP BS, when the metric thickness of the $\mathrm{SiO}_{2}$ film is kept constant (d $=162.8 \mathrm{~nm}$ ).

Other pairs of film and substrate refractive indices $n_{1}<n_{2}$ were tried. In general, the angular range over which Eq. (6) is satisfied is much more restricted, and the results are inferior to those presented in Section 3 for systems for which $n_{1}>n_{2}$.

\section{Summary}

We have described the systematic design of a singlelayer-coated BS for DOAP (Section 2). The BS consists of a high-index film on a low-index prismatic substrate (Fig. 2). The design procedure is based on satisfying the optimum phase (delta) condition of Eq. (6) exactly while maximizing the normalized determinant of the instrument matrix, Eq. (10). Specific results are obtained for BSs by using several material systems, including $\mathrm{ZnS}$ and GaP thin films on silica in the visible and $\mathrm{Si}, \mathrm{Ge}$, and $\mathrm{PbTe}$ thin films on an Irtran 1 substrate in the infrared (Section 3). The angular and spectral response of the Ge/Irtran 1 infrared design is also given (Section 3). Use of a lowindex substrate $\left(n_{2}<1.5\right)$ makes the prism angle $\alpha$ (Fig. 2) fall within the reasonable range of $40^{\circ}$ to $55^{\circ}$.

Low-index films on high-index substrates can also be used as DOAP BSs but the determinant of the instrument of the matrix is, in general, lower (Section 4), and the required prism angle is small. A specific design uses a $16^{\circ} \mathrm{Si}$ prism (or wedge), which is coated with a $\mathrm{SiO}_{2}$ film on the beam-splitting face and a $\mathrm{Si}_{3} \mathrm{~N}_{4}$ antireflection layer on the exit face, at a $73^{\circ}$ angle of incidence and wavelength $\lambda$ $=1.55 \mu \mathrm{m}$.

Because multilayer coatings provide more degrees of freedom, it should be apparent that all of the optimum conditions of Eqs. (6)-(8) can be satisfied simultaneously with such coatings. DOAP BSs that function at lower angles of incidence (e.g., $45^{\circ}$ or $60^{\circ}$ ) are also possible. Such extensions of the present work fall outside the scope of this paper.

\section{References}

1. R. M. A. Azzam, "Ellipsometry," in Handbook of Optics, 2nd ed., M. Bass, E. W. van Stryland, D. R. Williams, and W. L. Wolfe, eds. (McGraw-Hill, New York, 1995), Vol. II, Chap. 27.

2. R. A. Chipman, "Polarimetry," in Handbook of Optics, 2nd ed., M. Bass, E. W. van Stryland, D. R. Williams, and W. L. Wolfe, eds. (McGraw-Hill, New York, 1995), Vol. II, Chap. 22.

3. P. S. Hauge, "Recent developments in instrumentation in ellipsometry," Surf. Sci. 96, 108-140 (1980).

4. R. M. A. Azzam, "Division-of-amplitude photopolarimeter (DOAP) for the simultaneous measurement of all four Stokes parameters of light," Opt. Acta 29, 685-689 (1982).

5. R. M. A. Azzam, "Beam splitters for the division-of-amplitude photopolarimeter," Opt. Acta 32, 1407-1412 (1985).

6. R. M. A. Azzam and A. G. Lopez, "Accurate calibration of the four-detector photopolarimeter with imperfect polarizing optical elements,” J. Opt. Soc. Am. A 6, 1513-1521 (1989).

7. K. Brudzewski, "Static Stokes ellipsometer: general analysis," J. Mod. Opt. 38, 889-896 (1991).

8. S. Krishnan, "Calibration, properties, and applications of the division-of-amplitude photopolarimeter at 632.8 and 1523 nm,” J. Opt. Soc. Am. A 9, 1615-1622 (1992).

9. F. Delplancke, "Automated high-speed Mueller matrix scatterometer," Appl. Opt. 36, 5388-5395 (1997).

10. E. Compain and B. Drevillon, "Broadband division-ofamplitude polarimeter based on uncoated prisms," Appl. Opt. 37, 5938-5946 (1998).

11. R. M. A. Azzam, "Recent developments of division-ofamplitude photopolarimeters," in Proceedings of the Interna- 
tional Symposium on Polarization Analysis and Applications to Device Technology, T. Yoshizawa and H. Yokota, eds., Proc. SPIE 2873, 1-4 (1996).

12. R. M. A. Azzam and N. M. Bashara, Ellipsometry and Polarized Light (North-Holland, Amsterdam, 1987).

13. R. M. A. Azzam and A. De, "Optimal beam splitters for the division-of-amplitude photopolarimeter," J. Opt. Soc. Am. A 20, 955-958 (2003).

14. W. L. Wolfe, "Properties of optical materials," in Handbook of
Optics, W. D. Driscoll and W. Vaughan, eds. (McGraw-Hill, New York, 1978), Sec. 7.

15. W. J. Tropf, M. T. Thomas, and T. J. Harris, "Properties of crystals and glasses," in Handbook of Optics, M. Bass, E. W. van Stryland, D. R. Williams, and W. L. Wolfe, eds. (McGrawHill, New York, 1995), Vol. II, Chap. 33.

16. G. Eisenstein and L. W. Stulz, "High quality antireflection coatings on laser facets by sputtered silicon nitride," Appl. Opt. 23, 161-164 (1984). 